\title{
Correlational investigation of air pollutant emissions and fuel consumption of motor vehicle in various dynamic conditions
}

\author{
Zdzisław C. ${ }^{1}$, Jakub L. ${ }^{2 *}$, Jacek B. ${ }^{3}$ and Piotr W. ${ }^{3}$ \\ ${ }^{1}$ Automotive Industry Institute, Department of Scientific Activities, Jagiellonska 55, Warsaw, 03-301, Poland \\ ${ }^{2}$ Warsaw University of Technology, Narbutta 84, Institute of Vehicles, Warsaw, 02-524, Poland \\ ${ }^{3}$ Automotive Industry Institute, Engine and Chassis Laboratory, Jagiellonska 55, Warsaw, 03-301, Poland \\ Received: 23/09/2018, Accepted: 20/06/2020, Available online: 06/07/2020 \\ *to whom all correspondence should be addressed: e-mail: jakub.lasocki@pw.edu.pl \\ https://doi.org/10.30955/gnj.002893
}

\section{Graphical abstract}

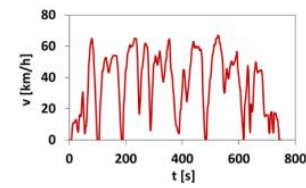

12 various driving tests

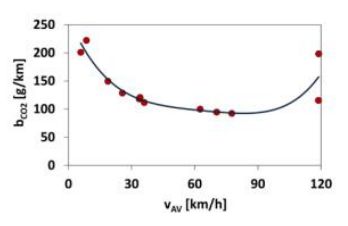

Characteristics of pollutant emission and fuel consumption

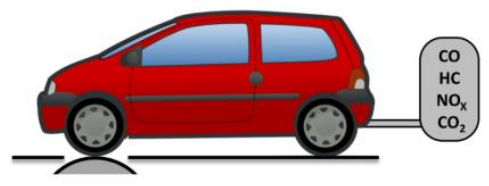

Vehicle testing on a chassis dynamometer $\checkmark$

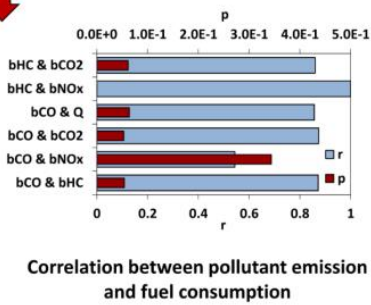

\section{Abstract}

Air pollutant emissions and fuel consumption of vehicles equipped with internal combustion engines are highly susceptible to the conditions of engine operation. The purpose of this research was to investigate the correlation between the emissions of individual pollutants (carbon monoxide, hydrocarbons, nitrogen oxides, and carbon dioxide), the fuel consumption and various dynamic conditions of the operation of an engine. The empirical data was obtained by testing of passenger car with a spark-ignition engine on a chassis dynamometer in 12 various driving tests, both type-approval and special. The results indicate, that the strongest correlation exists between the emissions of carbon dioxide and hydrocarbons and between the fuel consumption and the emissions of hydrocarbons and carbon dioxide. The weakest correlation was found to be between the emissions of carbon monoxide and nitrogen oxides. The average value of vehicle velocity proved to be suitable zero-dimensional characteristic of the dynamic driving conditions. The correlation between the emission of hydrocarbons and the average vehicle velocity can be assessed as the strongest, while between the emission of nitrogen oxides and the average vehicle velocity - the weakest.

Keywords: Pollutant emission, correlation, internal combustion engines, driving test cycles, exhaust emission.

\section{Introduction}

The operational properties of internal combustion (IC) engines are susceptible to the states of operation of the engines, i.e. not only to the static states but also, and above all at that, to the occurrence of dynamic states (Chłopek, 1999). This susceptibility is particularly distinct in the case of the emissions of pollutants harmful to human health; for the fuel consumption and carbon dioxide emission, the degree of this susceptibility is somewhat lower. Therefore, the results of examination of IC engine properties may only be considered with reference to the specific states of engine operation during the tests, which precludes any generalization of the examination results. In this connection, it is reasonable to investigate the susceptibility of the operational properties of IC engines to their states of operation, both static and dynamic. This objective may be accomplished by testing the properties of IC engines in the states determined by the engine operation conditions simulating the conditions of real operation of the engines. For automotive engines, such conditions are ensured by driving cycles simulating real vehicle movement.

For passenger cars and light-duty vehicles, many types of driving test cycles simulating driving in various traffic conditions have been developed. An important group of such tests consists of those used in type-approval procedures, e.g. New European Driving Cycle (NEDC) or Federal Test Procedure 75 (FTP-75) (Barlow et al., 2009). Apart from the type-approval tests, many other test cycles have been developed, usually intended to simulate vehicle drives in the time domain as accurately as possible. The example of such approach is Athens Driving Cycle (ADC-2002) (Tzirakis et al., 2006). To determine the distance specific pollutant emissions and distance specific fuel consumption in the software developed by INFRAS AG (2010), an extensive family of motor vehicle drive simulation tests has been prepared, which covers a very 
wide range of traffic conditions, from street congestions to high-speed (motorway) traffic (BUWAL and INFRAS AG, 1995; INFRAS AG, 2010). Within the implementation of the European Artemis program, a system of Common Artemis Driving Cycles has been prepared, which consists of test cycles that simulate motor car drives in various traffic conditions (André et al., 2009; Barlow et al., 2009). Another example of vehicle driving test cycles is the system of test cycles developed at a French institute INRETS (Institut National de Recherche sur les Transports et leur Sécurité, i.e. National Institute of Transport and Safety) (Nollet et al., 2000). At PIMOT (Automotive Industry Institute) in Warsaw, families of vehicle driving test cycles have been prepared (Chłopek et al., 2013b), which simulate the conditions of motor car drives in street congestion, urban, extra-urban, and high-speed (motorway and expressway) traffic. The PIMOT tests are treated as realizations of stochastic processes that characterize the conditions of vehicle drives (Chłopek et al., 2013a, 2013b).

In this work, a task was undertaken to answer a question whether results of the testing of pollutant emissions and fuel consumption in specific states of operation of an IC engine provide grounds for drawing conclusions about these properties in other engine operation conditions. Obviously, the drawing of such conclusions in general terms is unreasonable, but there is a possibility of considering IC engine operation states that would be similar to each other, in a predefined sense. Such states were sought in the driving test cycles simulating the real operation of automotive engines in moving vehicles. In spite of fundamental differences between the driving test cycles under consideration, there are also some similarities between them, especially when the average vehicle velocity values are close to each other.

\section{Material and methods}

The empirical tests were carried out on a Honda Civic EJ9 car with a spark-ignition engine of $1396 \mathrm{~cm}^{3}$ capacity, meeting the requirements of the Euro 3 exhaust emission standard. The car was tested with its engine warmed up to a stable temperature.

The pollutant emission and fuel consumption tests were carried out with the use of the following type-approval test cycles (Barlow et al., 2009):

- NEDC (New European Driving Cycle), consisting of the UDC (Urban Driving Cycle) and EUDC (Extra Urban Driving Cycle) tests;

- FTP-75 (Federal Test Procedure);

- HWFET (Highway Fuel Economy Test);

- Japan 10-15 Mode;

special test cycles (BUWAL and INFRAS AG, 1995):

- "Stop-and-Go" test cycle, simulating a vehicle drive in street congestions;

- "Autobahn" test cycle, simulating a vehicle drive on motorways and expressways; and test cycles developed by Chłopek et al. (2013b), where passenger car drives in the following conditions were simulated:

- street congestions (denoted by "CT");

- urban traffic without congestions (denoted by "UT");

- extra-urban (rural) traffic (denoted by "RT");

- high-speed traffic (on motorways and expressways, denoted by "HT").

The car was subjected to each of the test cycles five times. Tests were carried out on a vehicle chassis dynamometer Schenk Komeg EMDY 48. To examine the pollutant emissions, an exhaust gas analysing test stand was used, which incorporated a Horiba Mexa 7200 system provided with Horiba analysers to measure the concentrations of carbon monoxide (AIA-721A), hydrocarbons (FIA-725A), nitrogen oxides (CLA-755A), carbon dioxide (AIA-722), and oxygen (MPA-720).

The correlational interdependences between the results of measurements of pollutant emissions and fuel consumption were investigated. The coefficient of Pearson's linear correlation (Pearson, 1904) and the probability that the hypothesis of absence of Pearson's linear correlation would not be rejected for the sets of results were calculated. This approach is often used in the analysis of correlation between atmospheric pollutants (Jiang et al., 2016; Lee et al., 2016).

\section{Results and discussion}

Figure 1 shows the average value of the distance specific pollutant emissions and the average value of the operational fuel consumption determined experimentally in individual test cycles.

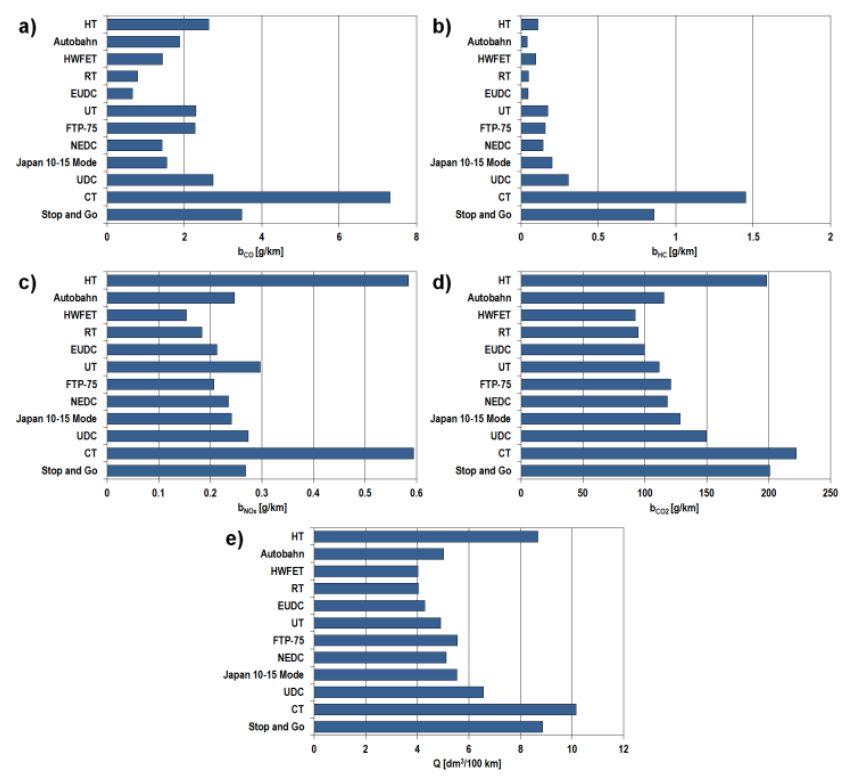

Figure 1. Average value of the distance specific emission of: a) carbon monoxide $-b_{\mathrm{CO}}, b$ ) hydrocarbons $\left.-b_{\mathrm{HC}}, c\right)$ nitrogen oxides $-b_{\mathrm{NO}}, d$ ) carbon dioxide $-b_{\mathrm{CO} 2}$ and e) average value of the operational fuel consumption $-b_{\mathrm{co}}$ in driving test cycles

The test results have indicated high susceptibility of pollutant emissions and fuel consumption to the conditions of vehicle motion in individual tests. Figure 2 shows the 
coefficient of variation for the sets of distance specific pollutant emissions and operational fuel consumption in individual tests. Especially for hydrocarbons, the susceptibility of distance specific emission of this pollutant to the state of engine operation in individual tests should be assessed as high.

The dependence of the average value of the distance specific pollutant emissions and the average value of the operational fuel consumption on the average vehicle velocity in individual tests has been presented in Figure 3.

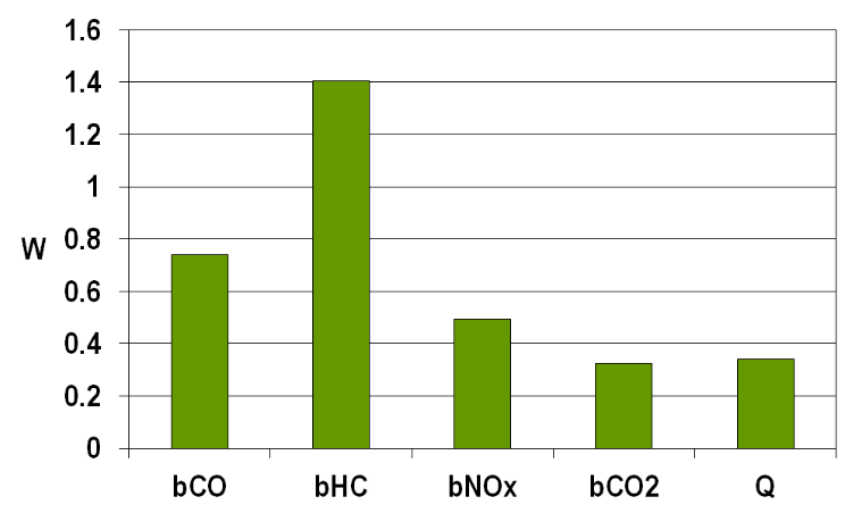

Figure 2. Coefficient of variation (W) for the sets of distance specific pollutant emissions and operational fuel consumption in individual driving test cycles
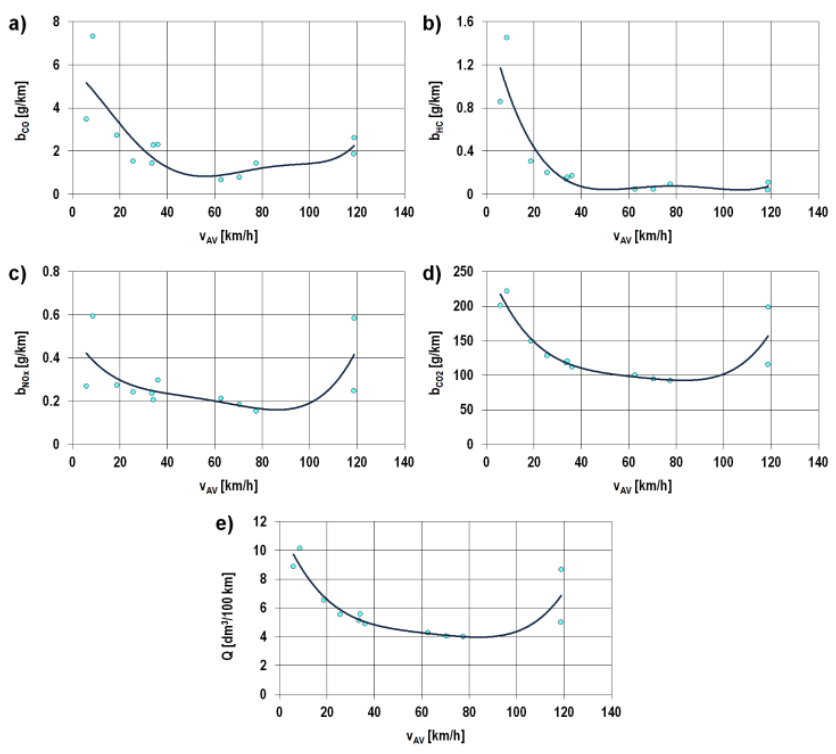

Figure 3. The dependence of distance specific emission of: a) carbon monoxide, b) hydrocarbons, c) nitrogen oxides, d) carbon dioxide, and e) operational fuel consumption on average vehicle velocity in driving test cycles

The general shape of the curves plotted is consistent with the current state of the knowledge, e.g. with the curves plotted by Xiaomeng et al. (2015).

Apart from the conclusion formulated previously about the significant susceptibility of test results to the conditions of vehicle motion in individual tests, it is noteworthy that even at similar average vehicle velocity in different tests, the test results may considerably differ from each other.
This confirms the hypothesis that the average vehicle velocity should not be taken as the only zero-dimensional parameter defining the dynamic states of the IC engine in a motor vehicle that determine the pollutant emissions and fuel consumption. Another parameter, supplementing the description of vehicle operation conditions, could be the average absolute value of the product of velocity and positive acceleration of the vehicle (BUWAL and INFRAS AG, 1995; Chłopek, 1999).

The correlational interdependences between pollutant emissions and fuel consumption in various dynamic conditions of IC engine operation, determined by the engine operation in various driving tests, were investigated with the use of sets of the test results presented.

The first investigations were carried out to verify the correlation between the sets of results of testing the distance specific pollutant emissions and the operational fuel consumption in various conditions of IC engine operation, determined by the vehicle motion in individual driving test cycles. The results of determining the coefficient of Pearson's linear correlation and the probability that the hypothesis of absence of Pearson's linear correlation would not be rejected for the sets under analysis have been presented in Figure 4. Figure $4 a$ and $4 b$ show the values determined for the test combinations for which the correlation was found to be the strongest; for the test combinations with the weakest correlation, the results have been shown in Figure $4 \mathrm{c}$ and $4 \mathrm{~d}$.
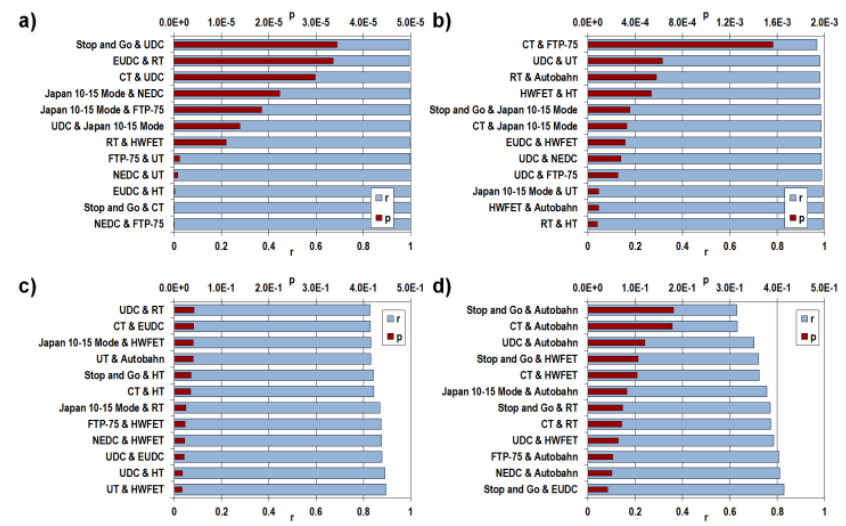

Figure 4. Coefficient of Pearson's linear correlation $(r)$ and probability that the hypothesis of absence of Pearson's linear correlation would not be rejected ( $p$ ) for the sets of results concerning the distance specific pollutant emissions and the operational fuel consumption in the test pairs: $a, b-$ test combinations with the strongest correlation; $c, d-$ test combinations with the weakest correlation

The correlation between the distance specific emission and the operational fuel consumption for dynamic states of operation of an IC engine for different pairs of the driving test cycles should be assessed as strong. The highest value of the probability that the hypothesis of absence of correlation would not be rejected for the sets under analysis is lower than 0.2. In general, the strongest correlation can be observed, as expected, between the sets of results of the tests in which the average vehicle velocity 
are close to each other, i.e. the tests representing similar vehicle drive conditions. This is the case e.g. for the test pairs NEDC \& FTP-75, Stop-and-Go \& CT, EUDC \& HT, or NEDC \& UT. The correlation was found to be the weakest for the tests where the drive conditions significantly differ from each other, e.g. for the test pairs Stop-and-Go \& Autobahn and CT \& Autobahn.

The second series of investigations was carried out to assess how strong interdependences exist between individual pollutant emissions and the operational fuel consumption in various IC engine operation conditions. The results of determining the coefficient of Pearson's linear correlation and the probability that the hypothesis of absence of Pearson's linear correlation would not be rejected for the sets under analysis have been presented in Figure 5.

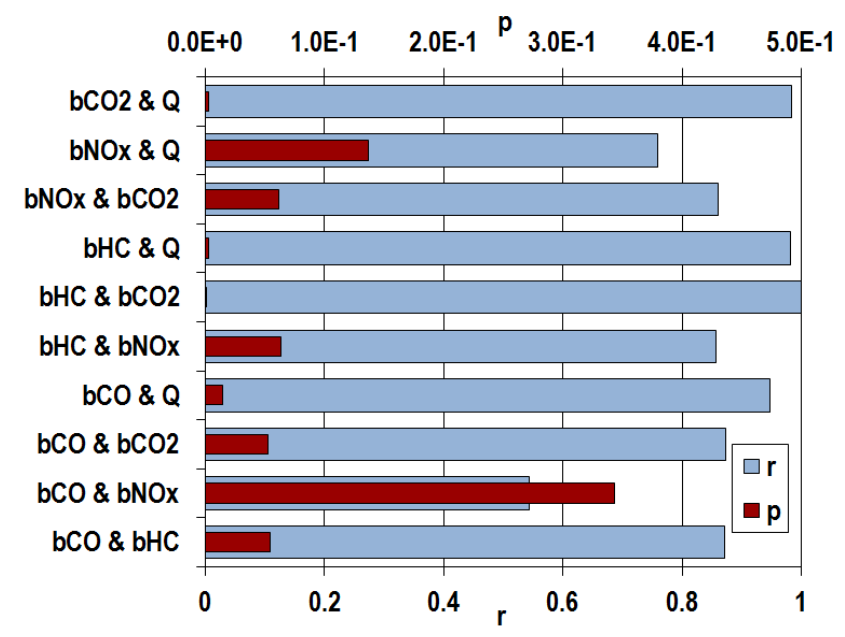

Figure 5. Coefficient of Pearson's linear correlation ( $r$ ) and probability that the hypothesis of absence of Pearson's linear correlation would not be rejected $(p)$ for individual combinations of results concerning the distance specific pollutant emissions and the operational fuel consumption in all the tests

The correlation between individual sets was found to be highly diverse. The strongest correlation was found to exist between the distance specific emission of carbon dioxide and the distance specific emission of hydrocarbons and between the operational fuel consumption and distance specific emissions of hydrocarbons and carbon dioxide. The weakest correlation was revealed to be between the distance specific emission of carbon monoxide and the distance specific emission of nitrogen oxides.

In consideration of some regularity of the dependence of distance specific pollutant emissions and operational fuel consumption on the average vehicle velocity, the correlational interdependences between the sets of distance specific pollutant emissions and operational fuel consumption values and the set of average velocity values were examined. The results of this examination have been presented in Figure 6.

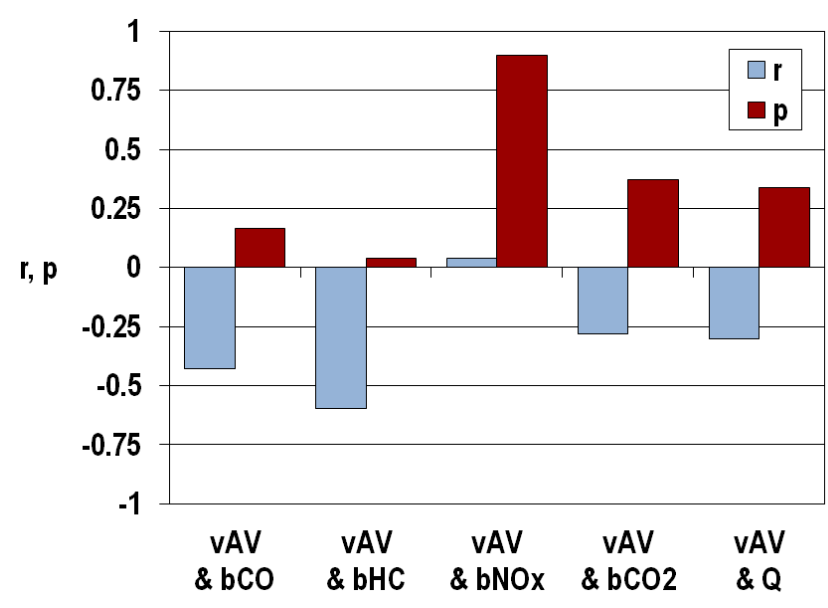

Figure 6. Coefficient of Pearson's linear correlation ( $r$ ) and probability that the hypothesis of absence of Pearson's linear correlation would not be rejected $(p)$ for individual combinations of results of determining the pollutant emissions and operational fuel consumption and the average vehicle velocity in all the tests

It was found that the strongest correlation existed between the distance specific emission of hydrocarbons and the average vehicle velocity and that the weakest correlation existed between the distance specific emission of nitrogen oxides and the average vehicle velocity. Based on these results, an opinion may be formulated that the dependence on the IC engine operation states is the strongest in the case of the distance specific emission of hydrocarbons and the weakest in the case of the distance specific emission of nitrogen oxides. This is confirmed, in the case of hydrocarbons, by the coefficient of variation for the sets of specific distance pollutant emissions and operational fuel consumption in individual tests (Figure 2).

\section{Conclusions}

The investigations carried out on pollutant emissions and fuel consumption in tests simulating diverse driving conditions of motor vehicle justify the statement that there are reasonable grounds for an objective assessment of the IC engine properties under examination in the conditions of various tests, based on the knowledge of results of tests carried out to other test procedures. Such a conclusion stems from the results of correlational investigations on the sets of results of testing the pollutant emissions and operational fuel consumption in the test cycles under consideration. This is particularly reasonable in the case of the test cycles that are characterized by similar values of the average vehicle velocity. This confirms that the average value of vehicle velocity is an effective zero-dimensional parameter describing specific states of operation of an automotive engine in respect of its operational properties (BUWAL and INFRAS AG, 1995; Chłopek, 1999).

Another conclusion drawn from the investigations carried out is that no considerable correlation has been found between the results of determining the distance specific pollutant emissions and the operational fuel consumption in various driving test cycles. 


\section{Acknowledgements}

This paper is based on results of tests carried out within research grant No. N N509556440 entitled "Sensitivity of pollutant emissions and fuel consumption to the conditions of operation of a spark-ignition engine", sponsored by the National Science Centre of Poland.

\section{References}

André M., Keller M., Sjödin Å., Gadrat M., McCrae I. and Dilara P. (2009), The Artemis European tools for estimating the transport pollutant emissions, Proceedings of the 18th International Emission Inventories Conference, April 14-17, 2009, Baltimore, Maryland, USA, 1-10.

Barlow T.J., Latham S., McCrae I.S. and Boutler P.G. (2009), A reference book of driving cycles for use in the measurement of road vehicle emission, Version 3. TRL Limited, United Kingdom.

BUWAL (Bundesamt für Umwelt, Wald und Landschaft), INFRAS AG (Infrastruktur- Umwelt- und Wirtschaftsberatung). (1995), Luftschadstoffemissionen des Strassenverkehrs 1950-2010, BUWAL-Bericht Nr. 255, Bern.

Chłopek Z. (1999), Modelling of exhaust emission processes in the conditions of operation of combustion engines in mobile applications, Publishing House of the Warsaw University of Technology, Warsaw.

Chłopek Z., Biedrzycki J., Lasocki J. and Wójcik P. (2013a), Investigation of pollutant emissions from a motor vehicle engine in tests simulating real vehicle use in road traffic conditions, Combustion Engines, 154, 202-207.

Chłopek Z., Biedrzycki J., Lasocki J. and Wójcik P. (2013b), Investigation of the motion of motor vehicles in Polish conditions, Archives of Automotive Engineering, 60, 3-20.

INFRAS AG (Infrastruktur- Umwelt- und Wirtschaftsberatung) (2010), Handbook emission factors for road transport 3.1, Quick reference, Version 3.1. Bern.

Jiang Z., Zeng Q., Pi H. and Tie B. (2016), Variation in characteristics of air concentrations of $\mathrm{NH}_{3}, \mathrm{NO}_{2}$ and $\mathrm{O}_{3}$ induced by applications of urea in soils of plastic greenhouses in suburban China, Atmospheric Pollution Research, 7, 619625.

Lee J.Y., Ryu S.H., Kim C.H. and Bae G.N. (2016), Indoor-to-outdoor pollutant concentration ratio modeling of $\mathrm{CO}_{2}, \mathrm{NO}_{2}$, and lungdeposited nanoparticles, Atmospheric Pollution Research, 7, 664-670.

Nollet V., Schadkowski C., Hue S., Flandrin Y. and Dechaux J.C. (2000), Elaboration d'un cadastre d'émissions de polluants primaires dans la region Nord-Pas-de-Calais. Les transports automobiles, Revue Pollution Atmosphérique, 165, 109-119.

Pearson K. (1904), On the theory of contingency and its relation to association and normal correlation, Dulau and Co., London.

Tzirakis E., Pitsas K., Zannikos F. and Stournas S. (2006), Vehicle Emissions and Driving Cycles: Comparison of the Athens Driving Cycle (ADC) with ECE-15 and European Driving Cycle (EDC), Global NEST Journal, 8, 282-290.

Xiaomeng W., Shaojun Z., Ye W., Zhenhua L., Wenwei K., Lixin F. and Jiming H. (2015), On-road measurement of gaseous emissions and fuel consumption for two hybrid electric vehicles in Macao, Atmospheric Pollution Research, 6, 858-866. 